\title{
Signal Model and Parameter Estimation for Colocated Mimo Radar
}

\author{
Moein Ahmadi*, Kamal Mohamed-pour† \\ K.N. Toosi University of Technology, Iran. \\ *moein@ee.kntu.ac.ir, †kmpour@kntu.ac.ir
}

Received: February 7, 2021. Revised: Novemeber 8, 2021. Accepted: December 20, 2021. Published: January 5, 2022.

\begin{abstract}
In this paper, we consider the signal model and parameter estimation for multiple-input multiple-output (MIMO) radar with colocated antennas on stationary platforms. Considering internal clutter motion, a closed form of the covariance matrix of the clutter signal is derived. Based on the proposed closed form and low rank property of the clutter covariance matrix and by using the singular value decomposition, we have proposed a subspace model for the clutter signal. Following the proposed signal model, we have provided maximum likelihood (ML) estimation for its unknown parameters. Finally, the application of the proposed ML estimation in space time adaptive processing (STAP) is investigated in simulation results. Our ML estimation needs no secondary training data and it can be used in scenarios with nonhomogeneous clutter in range.
\end{abstract}

Keywords: Multiple-input multiple-output (MIMO) radar, Clutter covariance matrix (CCM), Maximum likelihood (ML) estimation, Space time adaptive processing (STAP).

\section{INTRODUCTION}

Multiple-input multiple-output (MIMO) radar concept has received great attention in recent years (see [1]-[12]). MIMO radars emit multiple orthogonal probing waveforms through multiple transmit antennas and receive the echoes reflected by the target by multiple receive antennas. Based on the array configurations used, there are two types of MIMO radar. The first type employs widely separated transmit/receive antennas to capture the target's radar cross section diversity and detection diversity gain [3]. The second type of MIMO radar uses closely spaced transmit/receive antennas, which can provide higher spatial resolution [11], better parameter identifiability [12] and possibility of direct application of adaptive array techniques. In [16], the signal model and moving target detection for widely separated MIMO radar in spatially nonhomogeneous clutter have been considered.

In this paper, we focus on the latter type, henceforth referred to as colocated MIMO radar. We develop a signal model for colocated MIMO radar on a stationary platform. Our signal model includes the target, clutter and noise signals. The closed form of the clutter covariance matrix is derived without any secondary training data. For MIMO radars with space-time adaptive processing, one big concern is the clutter rank [15]. The low rank property of the derived closed form clutter covariance matrix is verified by computer simulations. We then develop a subspace model for the clutter signal which is based on singular value decomposition of clutter covariance matrix. The unknown parameters of the signal model are estimated by maximum likelihood estimation. The estimated clutter covariance matrix is calculated based on the received signal in one range bin of interest and requires no secondary training data. Therefore, the proposed signal model and parameter estimation can also be used in a scenario with nonhomogeneous clutter in range. We also investigate the application of proposed ML parameters estimation in MIMO STAP. In homogenous clutter environments, STAP needs $2 M N L$ secondary range bins to have an acceptable SINR performance. Thanks to the prior structure knowledge of the derived covariance matrices, an SINR performance that is close to SINR performance with perfect interference covariance matrix is achieved while there is no need to secondary data.

Matrices and vectors are denoted by uppercase and lowercase bold letters, respectively. $\mathbb{E}\{\},.(.)^{T}$ and $(.)^{H}$ stand for the statistical expectation, transpose and Hermitian transpose, respectively. Identity matrix and Kronecker product are represented by $I$ and $\Theta$, respectively.

The remainder of this paper is organized as follows. The signal model is introduced in Section 2. In Section 3, we propose the ML estimation of model's unknown parameters. Section 4 contains numerical results. Finally, conclusions are drawn in Section 5.

\section{SIGNAL MODEL}

Consider a colocated MIMO radar on a stationary platform with $M$ transmit antennas and $N$ receive antennas, as shown in Figure 1. The transmit and receive arrays are with $d_{T}$ and $d_{R}$ separation between two adjacent array elements, respectively. The $m$ th transmit antenna emits $\varphi_{m}(t), m=$ $0,1, \ldots, M-1$ which satisfies the orthogonal waveforms condition

$$
\int_{T_{p}} \varphi_{m}(t) \varphi_{m}^{*}(t) d t= \begin{cases}1 & m=m^{\prime} \\ 1 & m \neq m^{\prime}\end{cases}
$$

where $T_{P}$ is the radar pulse width. The signal received by the $n$th receive antenna can be written as 


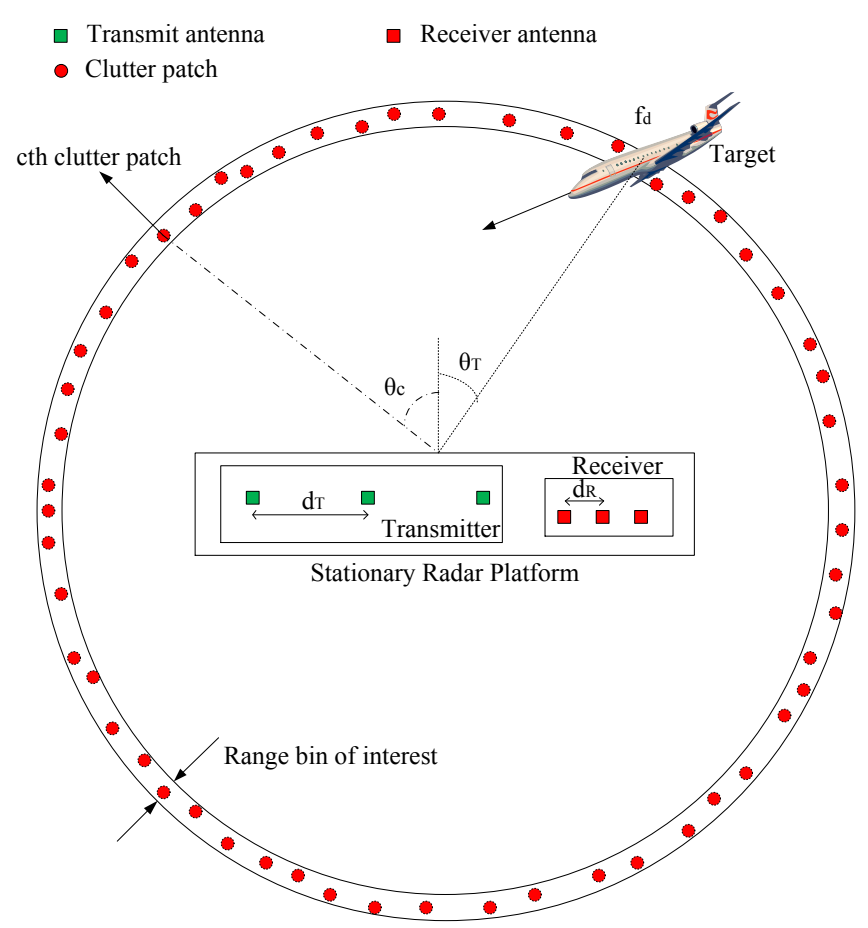

Figure 1: Colocated MIMO Radar

$$
\begin{gathered}
r_{n}(t)=\sum_{m=0}^{M-1} \alpha \sqrt{\frac{E}{M}} \varphi_{m}(t-\tau) e^{j 2 \pi f_{d} t} e^{j \frac{2 \pi}{\lambda}\left(m d_{T}+n d_{R}\right) \sin \theta_{T}} \\
+\sum_{i=0}^{N_{C}-1} \sum_{m=0}^{M-1} \alpha_{c, i} \sqrt{\frac{E}{M}} \varphi_{m}(t-\tau) e^{j 2 \pi f_{c, i} t} e^{j \frac{2 \pi}{\lambda}\left(m d_{T}+n d_{R}\right) \sin \theta_{c, i}} \\
+w_{n}(t) ; n=0,1, \ldots, N-1
\end{gathered}
$$

where $\alpha$ is the zero mean circular complex Gaussian amplitude of the signal reflected by the target, $E$ is the total energy of the transmitter, $\tau$ is the time delay of the range bin of interest, $f_{d}$ and $\theta_{T}$ are the Doppler frequency and the viewing direction of the target, respectively, $\lambda$ is the wavelength of the carrier signal, $N_{C}$ is the number of clutter patches, $\alpha_{c, i}$ is the zero mean circular complex Gaussian amplitude of the signal reflected by the $i$ th clutter patch, $f_{c, i}$ and $\theta_{c, i}$ are the Doppler frequency and the viewing direction of the $i$ th clutter patch. The internal clutter motions induced by, e.g., wind affecting a forest or grassland, make that clutter signal has Doppler frequency. It is assumed that $f_{c, i}, i=$ $1,2, \ldots, N_{C}$ are independent Gaussian random variables [14] and are also independent from their corresponding angles $\theta_{c, i}, i=1,2, \ldots, N_{C}$. The internal clutter motions limit the performance of radar in detection of targets with low radial velocity especially in low pulse repetition frequency radars. $w_{n}(t)$ is the complex Gaussian noise in the nth receive antenna output.

In a MIMO pulsed radar, the receive antennas collect $L$ pulses in a coherent processing interval (CPI). Each receive antenna separates components of the transmit signals using $M$ matched filters.

$$
\begin{aligned}
& x_{l, m, n}=\int_{T_{P}} r_{n}(t+l T) \varphi_{m}^{*}(t) d t \\
& =\alpha \sqrt{\frac{E}{M}} e^{j 2 \pi f_{d} l T} e^{j \frac{2 \pi}{\lambda}\left(m d_{T}+n d_{R}\right) \sin \theta_{T}} \\
& +\sum_{i=0}^{N_{C}-1} \alpha_{c, i} \sqrt{\frac{E}{M}} e^{j 2 \pi f_{c, i} l T} e^{j \frac{2 \pi}{\lambda}\left(m d_{T}+n d_{R}\right) \sin \theta_{c, i}}+ \\
& z_{l, m, n} ; l=0,1, \ldots, L-1 ; m=0,1, \ldots, M-1 ; \\
& n=0,1, \ldots, N-1 .
\end{aligned}
$$

where $T$ is the radar pulse repetition interval and $z_{l, m, n}$ are the samples of complex Gaussian noise. Stacking the samples, the $N M L$ vector is obtained as

$$
\mathbf{x}=\left[x_{0,0,0}, x_{0,0,1}, \ldots, x_{0,0, N-1}, \ldots, x_{L-1, M-1, N-1}\right]^{T} .
$$

The received vector can be expressed as

$$
\begin{aligned}
\mathbf{x}= & \alpha \sqrt{\frac{E}{M}} \mathbf{s}_{\text {MIMO }}\left(f_{d}, \theta_{T}\right)+ \\
& \sum_{i=0}^{N_{C}-1} \alpha_{c, i} \sqrt{\frac{E}{M}} \mathbf{s}_{M I M O}\left(f_{c, i}, \theta_{c, i}\right)+\mathbf{z} .
\end{aligned}
$$

where $\mathbf{s}_{\text {MIMO }}(f, \theta)=\mathbf{d}(f) \otimes \mathbf{a}(\theta) \otimes \mathbf{b}(\theta)$ is the MIMO steering vector and

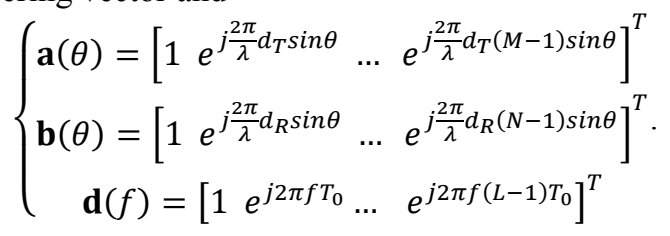

are the transmit and receive steering vector and Doppler steering vector, respectively.

STAP is a powerful approach to extract the signal of interest (SOI) in a spatially and frequency selective way in the presence of interferences such as clutter and noise. The main goal of an STAP algorithm is to maximize the signal to interference ratio (SINR). The output of a space-time adaptive processor is given by

$$
y=\mathbf{w}^{H} \mathbf{x} \text {. }
$$

where $\mathbf{w}$ is the vector of STAP weights. The optimum weight vector can be obtained by

$$
\mathbf{w}=\frac{1}{\mathbf{s}_{M I M O}^{H}\left(f_{d}, \theta_{T}\right) \mathbf{R}_{I}^{-1} \mathbf{s}_{M I M O}\left(f_{d}, \theta_{T}\right)} \mathbf{R}_{I}^{-1} \mathbf{s}_{M I M O}\left(f_{d}, \theta_{T}\right) .
$$

The covariance matrix $\mathbf{R}=\mathbb{E}\left\{\mathbf{x} \mathbf{x}^{H}\right\}$ can be expressed as

$$
\mathbf{R}=\mathbf{R}_{T}+\mathbf{R}_{I}=\mathbf{R}_{T}+\mathbf{R}_{C}+\sigma_{z}^{2} \mathbf{I} \text {. }
$$

where $\mathbf{R}_{T}$ is the covariance matrix of the target signal, $\mathbf{R}_{I}$ is the interference covariance matrix, $\mathbf{R}_{C}$ is the clutter covariance matrix and $\sigma_{z}^{2}$ is the variance of the noise. The covariance matrix of the target signal can be expressed as

$$
\mathbf{R}_{T}=\frac{\sigma_{\alpha}^{2} E}{M} \mathbf{s}_{M I M O}\left(f_{d}, \theta_{T}\right) \mathbf{s}_{M I M O}^{H}\left(f_{d}, \theta_{T}\right) .
$$


where $\sigma_{\alpha}^{2}$ is the variance of the signal reflected by the target. To compute the covariance matrix of the clutter, we assume that $\alpha_{c, i} ; i=0,1, \ldots, N_{C}$ are zero mean independent random variables with variance $\sigma_{c}^{2}$, and the clutter patches are distributed uniformly in azimuth angle which means that $\theta_{c} \sim U(0,2 \pi)$ and Doppler of each clutter patch is a zero mean Gaussian variable with variance $\sigma_{f}^{2} . \alpha_{c, i}, f_{c, i}$ and $\theta_{c, i}$ are independent random variables, therefore

$\mathbf{R}_{C}=\frac{\sigma_{c}^{2} E}{M} \sum_{i=0}^{N_{C}-1} \mathbb{E}_{f, \theta_{c, i}}\left\{\mathbf{s}_{M I M O}\left(f_{c, i}, \theta_{c, i}\right) \mathbf{s}_{M I M O}^{H}\left(f_{c, i}, \theta_{c, i}\right)\right\}$

If $r_{i, j} ; i, j=0,1, \ldots, N M L-1$, is the value of element $i$ th row and $j$ th column of $\mathbf{R}_{C}$, then

$$
\begin{aligned}
& r_{n+m N}+l M N, n^{\prime}+m^{\prime} N+l^{\prime} M N=\frac{\sigma_{c}^{2} E}{M} \sum_{i=0}^{N_{C^{-1}}} \mathbb{E}_{f_{c, i}}\left\{e^{j 2 \pi f_{c, i}\left(l-l^{\prime}\right) T_{0}}\right\} \\
& \times \mathbb{E}_{\theta_{c, i}}\left\{e^{j \frac{2 \pi}{\lambda}\left(\left(m-m^{\prime}\right) d_{T}+\left(n-n^{\prime}\right) d_{R}\right) \sin \theta_{c, i}}\right\} .
\end{aligned}
$$

The covariance matrix of the clutter can be computed by some straightforward calculations:

$$
\begin{aligned}
& \mathbf{R}_{C}=\left[r_{n+m N+l M N, n^{\prime}+m^{\prime} N+l^{\prime} M N}\right]=\frac{N_{C} \sigma_{c}^{2} E}{M} \times \\
& {\left[e^{-2\left(\pi T \sigma_{f}\left(l-l^{\prime}\right)\right)^{2}} J_{0}\left(\frac{2 \pi}{\lambda}\left(\left(m-m^{\prime}\right) d_{T}+\left(n-n^{\prime}\right) d_{R}\right)\right)\right] .}
\end{aligned}
$$

where $J_{0}($.$) is the zeroth order Bessel function of the first$ kind.

Since the clutter covariance matrix usually has a small rank [15], despite of high clutter power, the clutter signal has a good signature in observation space and STAP can suppress interference signal effectively. In Figure 2, the rank of clutter covariance matrix versus total dimension of MIMO steering vector has been simulated based on the closed form in Equation (11). To obtain the rank of clutter covariance matrix, the threshold, 0.01 largest eigenvalue, is used. It is clear that the STAP pulse number in a CPI is greater than one; $L=1$ is plotted in Figure 2 as worst case. The low rank property is degraded by increasing $d_{T} / d_{R}$.

\section{PARAMETER ESTIMATION}

The clutter component of the received signal

$$
\mathbf{c}=\sum_{i=0}^{N_{C}-1} \alpha_{c, i} \sqrt{\frac{E}{M}} \mathbf{s}_{M I M O}\left(f_{c, i}, \theta_{c, i}\right)
$$

falls within a subspace which is expanded by the columns of a matrix $\mathbf{U} \in \mathbb{C}^{N M L \times K} ; K<N M L$, therefore the clutter signal can be expressed as a linear combination of the columns of the matrix $\mathbf{U}$ :

$$
\mathbf{c}=\mathbf{U} \rho
$$

where $\boldsymbol{\rho}$ denotes the $K \times 1$ unknown complex coefficient vector associated with the clutter component of the received signal.

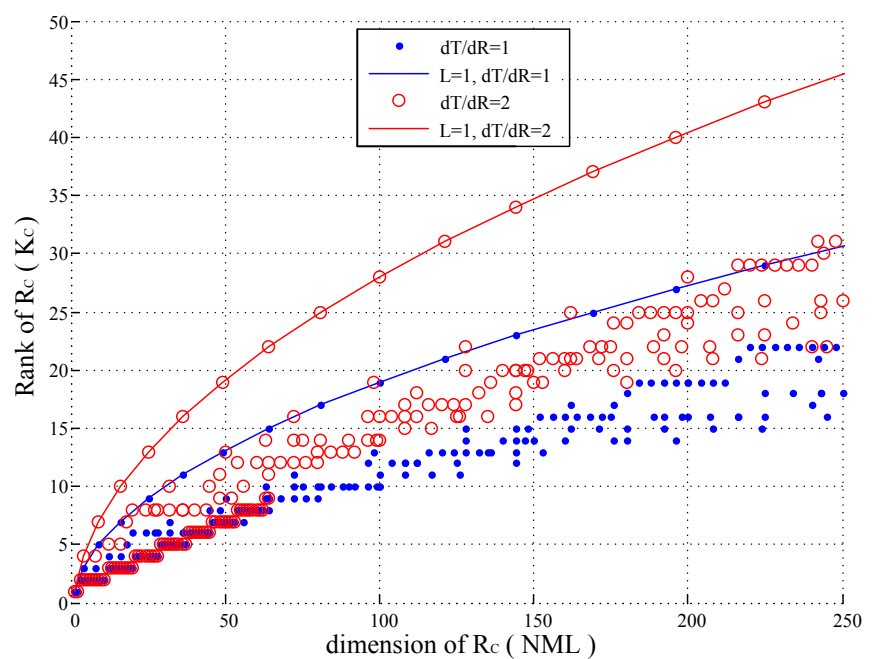

Figure 2: Rank of the covariance matrix of the clutter versus $\mathrm{NML}$ when $\mathrm{N}=\mathrm{M}$.

$\boldsymbol{R}_{C}$ can be expressed by singular value decomposition as

$$
\mathbf{R}_{C}=\mathbf{U}_{C} \boldsymbol{\Lambda}_{C} \mathbf{U}_{C}^{H} \text {. }
$$

where $\mathbf{U}_{C}$ and $\boldsymbol{\Lambda}_{C}$ are the eigenvector matrix and eigenvalue matrix, respectively. $\Lambda_{C}$ is a $N M L \times N M L$ diagonal matrix which has only $K_{C} \ll N M L$ significant elements. To determine the matrix $\boldsymbol{U}$, we can use the $K=K_{C}$ columns of $\mathbf{U}_{C}$ which are corresponding to the $K_{C}$ largest eigenvalues.

$$
\left\{\begin{array}{c}
{\left[\mathbf{U}_{C} \boldsymbol{\Lambda}_{C}\right]=\mathbf{s v d}\left(\mathbf{R}_{C}\right)} \\
\mathbf{U}=\mathbf{U}_{C}(:, 1: K)
\end{array} .\right.
$$

The MIMO radar signal can be modelled as

$$
\mathbf{x}=\alpha \sqrt{\frac{E}{M}} \mathbf{s}_{\text {MIMO }}\left(f_{T}, \theta_{T}\right)+\mathbf{U} \boldsymbol{\rho}+\mathbf{z}
$$

We assume that $f_{d}$ and $\theta_{T}$ are reported to the radar by another radar system. The target parameter $\alpha$, clutter parameter $\rho$ and noise parameter $\sigma_{z}^{2}$ can be estimated from the received signal. According to the MIMO radar signal model, the loglikelihood function can be expressed as

$$
\begin{aligned}
& L\left(\mathbf{x} ; \alpha, f_{d}, \theta_{T}, \boldsymbol{\rho}, \sigma_{z}^{2}\right)=-\pi N M L \sigma_{z}^{2} \\
& \quad-\frac{1}{\sigma_{z}^{2}}\left\|\mathbf{x}-\alpha \sqrt{\frac{E}{M}} \mathbf{s}_{M I M O}\left(f_{d}, \theta_{T}\right)-\mathbf{U} \boldsymbol{\rho}\right\|^{2} .
\end{aligned}
$$

where $\|\mathbf{x}\|$ denotes the vector 2-norm.

The estimation of noise variance can be computed by

$$
\partial L\left(\mathbf{x} ; \alpha, f_{d}, \theta_{T}, \boldsymbol{\rho}, \sigma_{z}^{2}\right) / \partial \sigma_{z}^{2}=0 .
$$

which leads to

$\hat{\sigma}_{z}^{2}=\frac{1}{N M L}\left\|\mathbf{x}-\alpha \sqrt{\frac{E}{M}} \mathbf{s}_{M I M O}\left(f_{d}, \theta_{T}\right)-\mathbf{U} \boldsymbol{\rho}\right\|^{2}$.

Therefore,

$L\left(\mathbf{x} ; \alpha, f_{d}, \theta_{T}, \boldsymbol{\rho}, \hat{\sigma}_{z}^{2}\right)=-N M L-$

$N M L l n\left(\frac{\pi}{N M L}\left\|\mathbf{x}-\alpha \sqrt{\frac{E}{M}} \mathbf{s}_{M I M O}\left(f_{d}, \theta_{T}\right)-\mathbf{U} \boldsymbol{\rho}\right\|^{2}\right)$. 


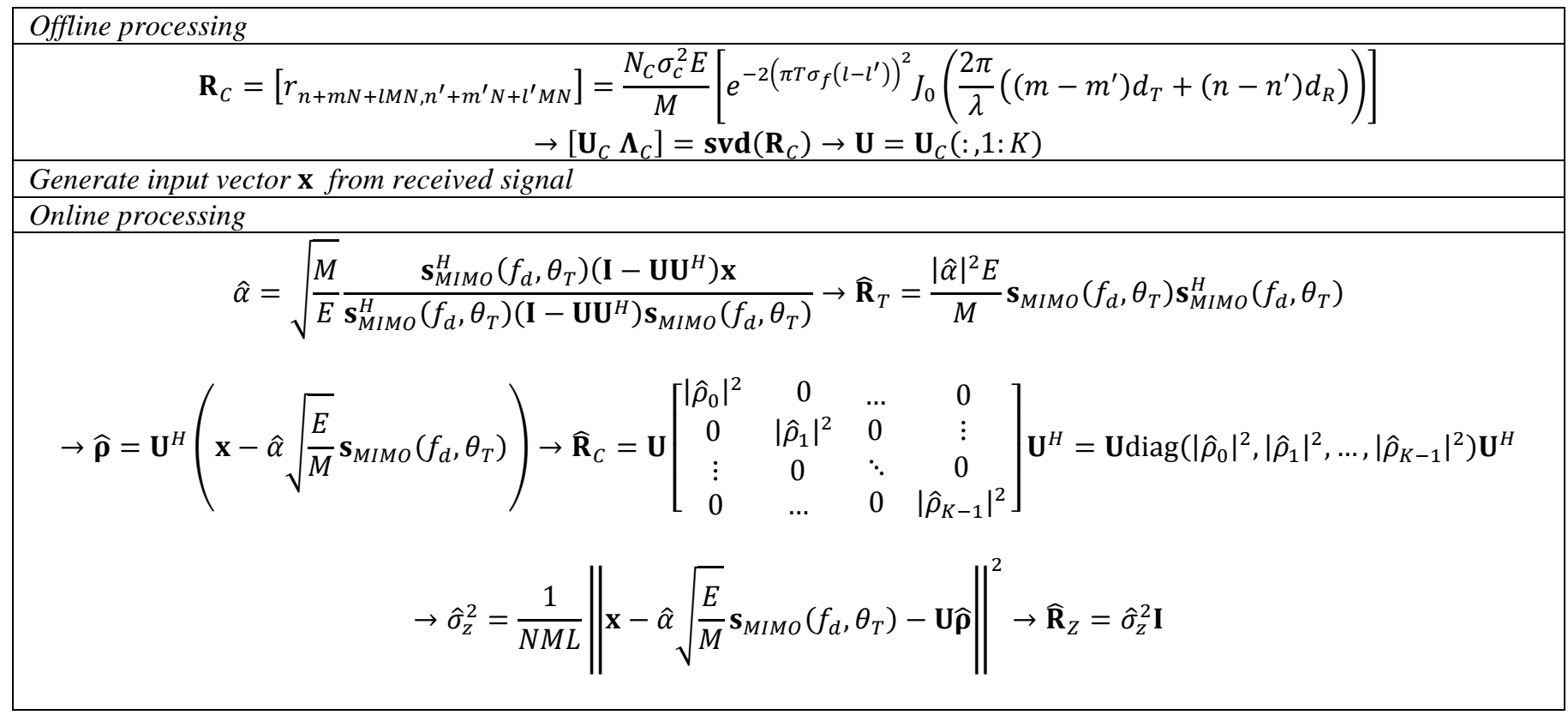

Table 1: Summary of offline and online processing of proposed model parameter estimation.

To maximize $L\left(\mathbf{x} ; \alpha, f_{d}, \theta_{T}, \boldsymbol{\rho}, \hat{\sigma}_{z}^{2}\right)$, the following cost function can be minimized

$C_{1}\left(\mathbf{x} ; \alpha, f_{d}, \theta_{T}, \boldsymbol{\rho}\right)=\left\|\mathbf{x}-\alpha \sqrt{\frac{E}{M}} \mathbf{s}_{M I M O}\left(f_{d}, \theta_{T}\right)-\mathbf{U} \boldsymbol{\rho}\right\|^{2}$

The ML estimation of $\rho$ is given by

$$
\widehat{\boldsymbol{\rho}}=\mathbf{U}^{H}\left(\mathbf{x}-\alpha \sqrt{\frac{E}{M}} \mathbf{s}_{M I M O}\left(f_{d}, \theta_{T}\right)\right) .
$$

Substituting the above estimation back into the cost function, we have

$$
\begin{aligned}
& C_{1}\left(\mathbf{x} ; \alpha, f_{d}, \theta_{T}, \widehat{\boldsymbol{\rho}}\right)=\left(\mathbf{x}-\alpha \sqrt{\frac{E}{M}} \mathbf{s}_{M I M O}\left(f_{d}, \theta_{T}\right)\right)^{H} \\
&\left(\mathbf{I}-\mathbf{U U}^{H}\right)\left(\mathbf{x}-\alpha \sqrt{\frac{E}{M}} \mathbf{s}_{M I M O}\left(f_{d}, \theta_{T}\right)\right) .
\end{aligned}
$$

Therefore, the ML estimate of $\alpha$ is given by

$$
\hat{\alpha}=\sqrt{\frac{M}{E}} \frac{\mathbf{s}_{M I M O}^{H}\left(f_{d}, \theta_{T}\right)\left(\mathbf{I}-\mathbf{U U} \mathbf{U}^{H}\right) \mathbf{x}}{\mathbf{s}_{M I M O}^{H}\left(f_{d}, \theta_{T}\right)\left(\mathbf{I}-\mathbf{U U} \mathbf{U}^{H}\right) \mathbf{s}_{M I M O}\left(f_{d}, \theta_{T}\right)} .
$$

The estimation of MIMO radar model parameters can be summarized as Table 1.

\section{SIMULATION RESULTS}

In this section, we use numerical results to demonstrate the performance of the proposed ML parameter estimation in STAP. STAP processor requires knowledge of the signal covariance matrix. In practice, it can be estimated from secondary data. The famous Reed-Mallet-Bernnan (RMB) result states that STAP needs approximately double overall dimension $(2 M N L)$ sufficiently homogeneous secondary range bins to achieve $3 \mathrm{~dB}$ SINR losses performance [13]. This large number of secondary data may not exist, especially in MIMO radar. In nonhomogeneous clutter environments, there are no sufficiently homogeneous secondary range bins.

Using the proposed ML estimation and matrix inversion lemma, the inverse interference covariance can be estimated as follows

$\widehat{\mathbf{R}}_{I}^{-1}=\left(\widehat{\mathbf{R}}_{C}+\hat{\sigma}_{z}^{2} \mathbf{I}\right)^{-1}=$

$\frac{1}{\widehat{\sigma}_{Z}^{2}}\left(\mathbf{I}-\mathbf{U d i a g}\left(\frac{\left|\widehat{\rho}_{0}\right|^{2}}{\left|\widehat{\rho}_{0}\right|^{2}+\widehat{\sigma}_{Z}^{2}}, \frac{\left|\hat{\rho}_{1}\right|^{2}}{\left|\widehat{\rho}_{1}\right|^{2}+\widehat{\sigma}_{Z}^{2}}, \ldots, \frac{\left|\hat{\rho}_{K-1}\right|^{2}}{\left|\widehat{\rho}_{K-1}\right|^{2}+\widehat{\sigma}_{Z}^{2}}\right) \mathbf{U}^{H}\right.$.

We assume a uniform linear array of $M=1,2,4$ transmit and $N=4$ receive omnidirectional antennas spaced half a wave length apart from each other which collect $L=8$ pulses in a CPI. The root-mean square of the clutter Doppler frequency $\sigma_{f}$ is assumed to be $60 \mathrm{~Hz}$, while the pulse repetition frequency is $3000 \mathrm{~Hz}$. Figure 3 shows the SINR performance of the STAP for SIMO and MIMO systems while their transmit powers are equal. The SINR of the STAP with perfect knowledge of covariance matrices is also plotted as a benchmark. The MIMO STAP with $M=4$ transmit antennas outperforms the SIMO STAP which has $M=1$ transmit antenna with four times power or equivalently four antennas with same power as MIMO antennas.

The MIMO system has more degree of freedom to suppress the interferences and therefore better SINR performance. In sample based covariance matrix estimation, it needs $2 N M L$ secondary range bins to have an acceptable SINR performance. In our approach, the SINR performance which is close to that with perfect interference covariance matrix is achieved while there is no need to use secondary data. This is because we have used the prior structures knowledge of covariance matrices which have been shown in Table 1, and 


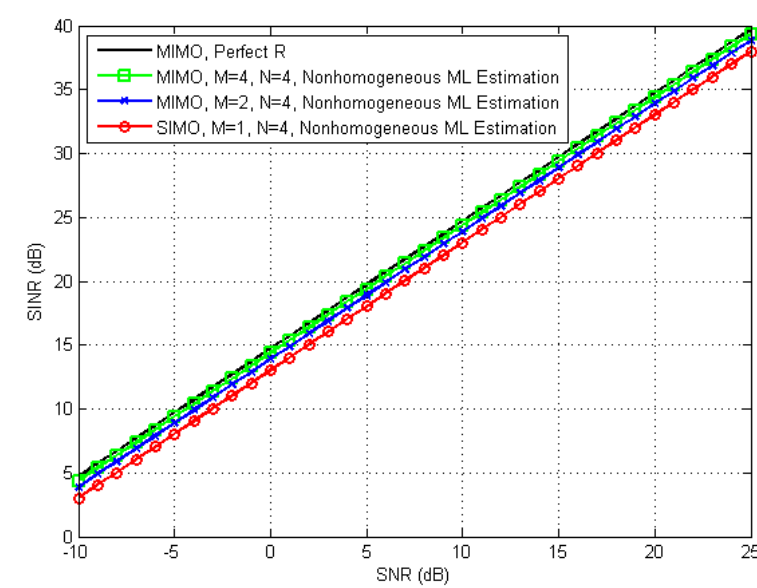

Figure 3: STAP SINR versus SNR for MIMO and SIMO radars with clutter to noise ratio $40 \mathrm{~dB}$.

we estimate the unknown parameters instead of the whole covariance matrix.

\section{CONCLUSION}

A signal model and maximum likelihood estimation of model parameters for MIMO radar with colocated antennas have been proposed. The proposed MIMO radar signal model includes the target, clutter and noise signals. The clutter covariance matrix has been derived analytically and based on its singular value decomposition, a subspace model for clutter signal has been proposed. Simulations have confirmed the low rank property of the extracted clutter covariance matrix. Maximum likelihood estimation for target amplitude, noise variance and clutter coefficients has been obtained with no need of secondary training data, and hence it can be used in the scenarios of nonhomogeneous clutter in range. Finally, ML estimation of environment parameters is used to obtain covariance matrix of interference for MIMO STAP application. Simulation results show that MIMO radar outperforms traditional SIMO radar in SINR performance.

\section{ACKNOWLEDGEMENTS}

This research was partly supported by Research Institute for ICT.

\section{REFERENCES}

[1] D. W. Bliss and K. W. Forsythe, Multiple-input multiple-output (MIMO) radar and imaging: degrees of freedom and resolution", Proceeding of 37th Asilomar Conference, volume 1, pp. 54-59, (2003).

[2] C. Y. Chong, F. Pascal, J.-P. Ovarlez, and M. Lesturgie, MIMO radar detection in non-Gaussian and heterogeneous clutter", IEEE Journal Selected Topics on Signal Processing, volume 4, no. 1, pp. 115-126, (2010).
[3] E. Fishler, A. M . Haimovich, R. S. Blum, L. J. Cimini, Jr, D. Chizhik, R. A. Valenzuela, -Sptial diversity in radars-models and detection performance", IEEE Transaction on Signal Processing, volume 54, no. 3, pp. 823-838, (2006).

[4] D. R. Fuhrmann and G. S. Antonio, Transmit beamforming for MIMO radar systems using signal cross-correlation", IEEE Transaction on Aerospace Electronic System, volume 44, no. 1, pp. 171-186, (2008).

[5] H. Godrich, A. Haimovich, and R. Blum, Farget localization accuracy gain in MIMO radar-based systems", IEEE Transaction on Information Theory, volume 56, no. 6, pp. 2783-2803, (2010).

[6] A. M. Haimovich, R. S. Blum, and L. J. Cimini, -MMO radar with widely separated antennas", IEEE Signal Processing Magazine, volume 25, no. 1, pp. 116-129, (2008).

[7] H. He, P. Stoica, and J. Li, Đesigning unimodular sequence sets with good correlations Including an application to MIMO radar", IEEE Transaction on Signal Processing, volume 57, no. 11, pp. 4391-4405, (2009).

[8] Q. He, R. Blum, H. Godrich, and A. Haimovich, Farget velocity estimation and antenna placement for MIMO radar with widely separated antennas", IEEE Journal Selected Topics on Signal Processing, volume 4, no. 1, pp. 79-100, (2010).

[9] Q. He, N. H. Lehmann, R. S. Blum, and A. M. Haimovich, MIMO radar moving target detection in homogeneous clutter", IEEE Transaction on Aerospace Electronic System, volume 46, no. 3, pp. 1290-1301, (2010).

[10] H. Li and B. Himed, - Transmit subaperturing for MIMO radars with co-located antennas", IEEE Journal Selected Topics on Signal Processing, volume 4, no. 1, pp. 5565, (2010).

[11] J. Li and P. Stoica, MIMO radar with colocated antennas", IEEE Signal Processing Magazine, volume 24, no. 5, pp. 106-114, (2007).

[12] J. Li, P. Stoica, L. Xu, and W. Roberts, -n parameter identifiability of MIMO radar", IEEE Signal Processing Letter, volume 14, no. 12, pp. 968-971, (2007).

[13] I. Reed, J. Mallett, L. Brennan, -Rajed convergence rate in adaptive arrays", IEEE Transaction on Aerospace and Electronic Systems, volume 10, no. 6, pp. 853-863, (1974).

[14] M. Skolnik, Introduction to Radar Systems (2nd ed.) Columbus, McGraw-Hill, 1980.

[15] G. Wang and Y. Lu, -Gitter Rank of STAP in MIMO Radar With Waveform Diversity", IEEE Transaction on Signal Processing, volume 58, no. 2, pp. 938-943, (2010).

[16] P. Wang, H. Li and B. Himed, Moving Target Detection Using Distributed MIMO Radar in Clutter With Nonhomogeneous Power", IEEE Transaction on Signal Processing, volume 59, no. 10, pp. 4809-4820, (2011).

\section{Creative Commons Attribution License 4.0 (Attribution 4.0 International, CC BY 4.0)}

This article is published under the terms of the Creative Commons Attribution License 4.0 https://creativecommons.org/licenses/by/4.0/deed.en_US 